\title{
Statistical Markup Language
}

National Cancer Institute

\section{Source}

National Cancer Institute. Statistical Markup Language. NCI Thesaurus. Code C47842.

Statistical markup language (StatML) is an xml specifically designed for exchange of statistical data. 